\title{
ON THE GESELOWITZ FORMULA IN BIOMAGNETICS
}

\author{
$\mathrm{BY}$ \\ GEORGE DASSIOS AND FOTINI KARIOTOU
}

University of Patras, Division of Applied Mathematics, Department of Chemical Engineering and ICE/HT-FORTH, Patras, Greece

\begin{abstract}
A detailed analysis of the Geselowitz formula for the magnetic induction and for the electric potential fields, due to a localized dipole current density, is provided. It is shown that the volume integral, which describes the contribution of the conductive tissue to the magnetic field, exhibits a hyper-singular behaviour at the point where the dipole source is located. This singularity is handled both via local regularization of the volume integral as well as through calculation of the total flux it gencrates. The analysis reveals that the contribution of the primary dipole to the volume integral is equal to the one third of the magnetic field generated by the primary dipole while the rest is due to the distributed conductive tissue surrounding the singularity. Furthermore, multipole expansion is introduced, which expresses the magnetic field in terms of polyadic moments of the electric potential over the surface of the conductor.
\end{abstract}

1. Introduction. Biomagnetism deals with the causal relation that connects electric currents generated within the organs of the human body with the magnetic fields they produce just outside the body $[7,11]$. Considering the size of human organs, such as the brain, the heart, or the lungs, the quasistatic approximation of Maxwell's equations for the investigation of these problems is well justified [7].

In the last three decades a significant number of papers have been written, both at the theoretical as well as the experimental level, which seek ways to determine the magnetic field for given currents inside a human organ (Direct Biomagnetic Problem), or to characterize the currents within the organ from measured extcrior niagnetic ficlds (Inverse Biomagnetic Problem). An excellent review on the subject is given by Hämäläinen et al. [7], where an extensive bibliography is also included. An interesting perturbation analysis on the shape of the conductor can be found in [12].

As far as theoretical models are concerned, almost all this work is based on the Geselowitz formula $[4,5]$ which relates the magnetic induction field in the exterior of the body to the actual electrochemically-generated currents and to the equivalent surface current distributions supported on surfaces scparating regions of different conductivity.

Received November 19, 2001 and, in revised form, March 1, 2002.

2000 Mathematics Subject Classification. Primary 78A25. 
The most tractable case is that for which the localized electrie activity of the neurons is represented by an equivalent electric dipole with a specified dipole moment. The human organ is considered to be a finite mion of regions of constant conductivity. One of these regions includes the primary souree. i.e.. the electric dipole. The primary source excites conduction currents to all component regions that have nonzero conductivity. These conduction currents are interpreted as volume distributions of dipole currents with moments that are proportional to the electric field. As Geselowitz has shown [5]. some vector identities combined with the vector invariant form of the dyadic version of Gauss s theorem lead to an equivalent expression for the magnetic field. where the dipoles are distributed over the boundaries that form the support of the discontinuities of the conductivity function for the particular organ. The moments of these surface distributions of dipoles now depend on the electric potential field as well as on the conductivity-jumps across the surfaces of discontinnity. For all regions that are free from primary currents this procedure is obviously justified. But at least one of the regions includes the primary current (dipole source). since otherwise no electromagnetic activity would be present. When this technique is applied to regions that involve sources, the analysis is more delicate since for such regions the electric potential. and consequently the electric field, involve singularities in the region of integration and therefore Gauss's theorem is not immediately applicable.

A careful treatment of these integrals involves the exclusion of the singularities by deleting a small sphere centered at the singularity and a subsequent limiting process that lets the radius of the sphere go to zero. After we exclude the singular point. Gauss's theorem can be used and the surface integral over the surface of the little sphere, depending on the strength of the singularity: can provide a nonvanishing contribution. This flux measuring approach has been used in an early version of the present work which misled us to conclude that an extra term should be added to the Geselowitz formula. We would like to acknowledge though that first professor Guido Nolte and then professor Jukka Sarvas and his colleagues Dr. Matti Kajola and Dr. Matti Hämäläinen pointed out to us that the extra term we calculated cancels out from a corresponding contribution of the volume integral over the little sphere since the field is also singular there. In fact the field is hyper-singular there and its contribution to the volume integral has to be evaluated either through regularization [3, 9] or through the theory of generalized functions [3]. The final result shows that the contribution of the volume integral to the magnetic induction field is due to the distributed conductive tissue as well as to a concentrated point measure at the point where the dipole is located and this last contribution equals to the one third of the field due to the primary dipole source. Through an appropriate use of polyadic analysis we show how a multipole expansion can be obtained, where the contribution of the conductive tissue enters via the polyadic surface moments of the interior electric potential. It is actually shown that the zeroth order moment equals the electric moment of the primary dipole.

The mathematical formulation of the Biomagnetic problem is stated in Sec. 2 while the analysis of the Geselowitz formula is developed in Sec. 3. Section 4 deals with the multipole expansion of the electric potential as well as the magnetic field. Based on the Geselowitz formula Sarvas [13] has calculated the exact magnetic field outside a 
spherical conductor. For early work on the sphere we refer to Ilmoniemi et al. [8]. A relative discussion is contained in Sec. 5 .

As it is well-known. the actual human brain is shaped in the form of an ellipsoid with average scmiaxes equal to $6,6.5$, and $9 \mathrm{~cm}$. The solution of the direct and the inverse MEG problem in ellipsoidal geometry is currently under investigation by the authors.

2. Mathematical formulation of the biomagnetic problem. Let $G$ be a bounded (open) region in $\mathbb{R}^{3}$ having a $C^{(1)}$-smooth boundary denoted by $S$. The unit normal $\hat{\mathbf{n}}$ on $S$ is directed towards the unbounded extcrior region $G_{0}=[G \cup S]^{c}$. Region $G$ represents a conductive human organ. such as the brain, while the conductivity of $G_{0}$ is assumed to be zero. A finite number of nonempty subregions $G_{1}, G_{2}, \ldots G_{n}$ divide $G$ in the sense that the union of their closures covers $G$,

$$
G_{i} \cap G_{j}=\varnothing
$$

for each $i, j=1,2, \ldots, n$ with $i \neq j$ and

$$
G_{i} \cap G_{0}=\varnothing . \quad i=1,2 \ldots n .
$$

Let

$$
S_{i j}=\bar{G}_{i} \cap \bar{G}_{j}, \quad i, j=1,2, \ldots, n
$$

where the bar denotes the topological closure, the common boundary separating the subregions $G_{i}, G_{j}$, and let $\hat{\mathbf{n}}_{i j}$ be the unit normal on $S_{i j} \operatorname{directed~from~} G_{i}$ to $G_{j}$. It is understood that the common boundary of non-neighbouring subregions is empty, and that each non-empty surface $S_{i j}$ is $C^{(1)}$-smooth. Furthermore. let

$$
S_{i 0}=\bar{G}_{i} \cap \bar{G}_{0}, \quad i=1,2 \ldots, n
$$

be the part of the $S$ boundary that is common to the $G_{i}$ subregion. The surface

$$
S_{i}=\bigcup_{j=1}^{n} S_{i j}
$$

describes the boundary of the subregion $G_{i}$, for each $i=1,2 \ldots n$.

The electric conductivity in each subregion $G_{i}$ is assumed to be constant and it is denoted by $\sigma_{i}, i=1,2, \ldots, n$. The exterior region $G_{0}$ has vanishing conductivity. The conductivity jumps occur on the separating surfaces $S_{i j}$ and $S_{i 0}$.

One of the subregions, let us say $G_{n}$, contains the primary dipole current in its interior $[6,10]$. That is,

$$
\mathbf{J}^{P}(\mathbf{r})=\mathbf{Q} \delta\left(\mathbf{r}-\mathbf{r}_{0}\right), \quad \mathbf{r}_{0} \in G_{n},
$$

where $\mathbf{Q}$ stands for the electric dipole moment and $\delta$ denotes the Dirac measure at $\mathbf{r}_{0}$. Within the realm of quasistatic electromagnetism [7] governed by the equations

$$
\begin{gathered}
\mathbf{E}(\mathbf{r})=-\nabla u(\mathbf{r}), \\
\nabla \times \mathbf{B}(\mathbf{r})=\mu_{0} \mathbf{J}(\mathbf{r}), \\
\mathbf{J}(\mathbf{r})=\mathbf{J}^{P}(\mathbf{r})+\sigma \mathbf{E}(\mathbf{r}), \\
\nabla \cdot \mathbf{B}(\mathbf{r})=0,
\end{gathered}
$$


the localized dipole current (6) gives rise to the electric potential

$$
u^{P}(\mathbf{r})=\frac{1}{4 \pi \sigma_{i}} \mathbf{Q} \cdot \frac{\mathbf{r}-\mathbf{r}_{0}}{\left|\mathbf{r}-\mathbf{r}_{0}\right|^{3}}, \quad \mathbf{r} \in G_{i}, \quad \mathbf{r} \neq \mathbf{r}_{0}
$$

and to the magnetic induction field

$$
\mathbf{B}^{P}(\mathbf{r})=\frac{\mu_{0}}{4 \pi} \mathbf{Q} \times \frac{\mathbf{r}-\mathbf{r}_{0}}{\left|\mathbf{r}-\mathbf{r}_{0}\right|^{3}}, \quad \mathbf{r} \neq \mathbf{r}_{0},
$$

where $\sigma_{i}$ denotes the conductivity of the subregion $G_{i}$ and $\mu_{0}$ denotes the magnetic permeability which is taken to be constant both in the space inside and outside the conductor. Equations (8), (10), and Gauss's theorem imply Ampère's law [10. 13]:

$$
\mathbf{B}(\mathbf{r})=\frac{\mu_{0}}{4 \pi} \int_{G} \mathbf{J}\left(\mathbf{r}^{\prime}\right) \times \frac{\mathbf{r}-\mathbf{r}^{\prime}}{\left|\mathbf{r}-\mathbf{r}^{\prime}\right|^{3}} d \nu\left(\mathbf{r}^{\prime}\right),
$$

where the integration is extended over the conductive region $G$. Relations (6), (7), (9), and the partition of $G$ in subregions of constant conductivity allow for (13) to be written as

$$
\mathbf{B}(\mathbf{r})=\frac{\mu_{0}}{4 \pi} \mathbf{Q} \times \frac{\mathbf{r}-\mathbf{r}_{0}}{\left|\mathbf{r}-\mathbf{r}_{0}\right|^{3}}-\frac{\mu_{0}}{4 \pi} \sum_{i=1}^{n} \sigma_{i} \int_{G_{i}} \nabla u_{i}\left(\mathbf{r}^{\prime}\right) \times \frac{\mathbf{r}-\mathbf{r}^{\prime}}{\left|\mathbf{r}-\mathbf{r}^{\prime}\right|^{3}} d \nu\left(\mathbf{r}^{\prime}\right),
$$

where the electric potentials $u_{i}, i=1,2, \ldots, n$ solve the following system of mixed boundary value problems:

$$
\begin{aligned}
\sigma_{i} \Delta u_{i}(\mathbf{r}) & =\nabla \cdot \mathbf{J}^{P}(\mathbf{r}), & & \mathbf{r} \in G_{i} \\
u_{i}(\mathbf{r}) & =u_{j}(\mathbf{r}), & & \mathbf{r} \in S_{i j} \\
\sigma_{i} \frac{\partial}{\partial n} u_{i}(\mathbf{r}) & =\sigma_{j} \frac{\partial}{\partial n} u_{j}(\mathbf{r}), & & \mathbf{r} \in S_{i j} \\
\frac{\partial}{\partial n} u_{i}(\mathbf{r}) & =0 . & & \mathbf{r} \in S_{i 0}
\end{aligned}
$$

for each $i=1,2, \ldots, n$. This is a well-posed problem (uniqueness holds up to an additive constant) that provides a solution in $G$. Once this problem is solved and the values of $u$ on $S$ are determined, the electric potential $u_{0}$ in the exterior $G_{0}$ is obtained as the solution of the Dirichlet problem

$$
\begin{aligned}
\Delta u_{0}(\mathbf{r}) & =0, & & \mathbf{r} \in G_{0} \\
u_{0}(\mathbf{r}) & =f(\mathbf{r}), & & \mathbf{r} \in S \\
u_{0}(\mathbf{r}) & =0\left(r^{-1}\right), & & r \rightarrow \infty
\end{aligned}
$$

where $f$ is composed of the traces of $u_{i}$ on $S_{i 0}$ for each $i=1.2, \ldots, n$. Because of (6), Eq. (15) becomes Laplace's equation for each $i=1,2, \ldots, n-1$, while for $i=n$, it is a Poisson's equation with a particular solution given by

$$
u_{n}^{P}(\mathbf{r})=u^{P}(\mathbf{r})=\frac{1}{4 \pi \sigma_{n}} \mathbf{Q} \cdot \frac{\mathbf{r}-\mathbf{r}_{0}}{\left|\mathbf{r}-\mathbf{r}_{0}\right|^{3}}, \quad \mathbf{r} \in G_{n} .
$$

Formula (14) expresses the magnetic induction field as the sum of the field generated by the primary source (6) at the point $\mathbf{r}_{0} \in G_{n}$ and the superposition of the fields due to a volume distribution of dipoles with moments given by

$$
\mathbf{E}_{i}\left(\mathbf{r}^{\prime}\right)=-\sigma_{i} \nabla u_{i}\left(\mathbf{r}^{\prime}\right), \quad \mathbf{r}^{\prime} \in G_{i} .
$$


Geselowitz's formula [4] concerns the replacement of these volume distributions with surface distributions of dipoles over the interfaces of changing conductivity. This is treated in the next section.

3. A study on the Geselowitz formula. Geselowitz's idea was to use Gauss's theorem to reduce the volume integrals in (14) to corresponding surface integrals and this was applied to all subregions that are free from primary current sources. The situation is more involved in the region that hosts the source and such a hosting region is always present. As Geselowitz [5] as well as his followers [2, 7, 12, 13] have claimed,

$$
\begin{aligned}
\nabla_{\mathbf{r}^{\prime}} u_{i}\left(\mathbf{r}^{\prime}\right) \times \frac{\mathbf{r}-\mathbf{r}^{\prime}}{\left|\mathbf{r}-\mathbf{r}^{\prime}\right|^{3}} & =\nabla_{\mathbf{r}^{\prime}} u_{i}\left(\mathbf{r}^{\prime}\right) \times \nabla_{\mathbf{r}^{\prime}} \frac{1}{\left|\mathbf{r}-\mathbf{r}^{\prime}\right|} \\
& =\nabla_{\mathbf{r}^{\prime}} \times\left[u_{i}\left(\mathbf{r}^{\prime}\right) \nabla_{\mathbf{r}^{\prime}} \frac{1}{\left|\mathbf{r}-\mathbf{r}^{\prime}\right|}\right]=\nabla_{\mathbf{r}^{\prime}} \times\left[u_{i}\left(\mathbf{r}^{\prime}\right) \frac{\mathbf{r}-\mathbf{r}^{\prime}}{\left|\mathbf{r}-\mathbf{r}^{\prime}\right|^{3}}\right] .
\end{aligned}
$$

where the fact that every gradient field is irrotational has been used.

In subregions free of sources the electric potential is harmonic and the vector invariant form of the dyadic Gauss's theorem [1],

$$
\int_{\Omega} \nabla \otimes \mathbf{f} d \nu=\int_{\partial \Omega} \hat{\mathbf{n}} \otimes \mathbf{f} d s
$$

with $\mathbf{f} \in C^{(1)}(\Omega) \cap C(\bar{\Omega})$ and $\Omega$ bounded with $C^{(1)}$-smooth boundary, imply that

$$
\int_{G_{i}} \nabla u_{i}\left(\mathbf{r}^{\prime}\right) \times \frac{\mathbf{r}-\mathbf{r}^{\prime}}{\left|\mathbf{r}-\mathbf{r}^{\prime}\right|^{3}} d \nu\left(\mathbf{r}^{\prime}\right)=\int_{S_{i}} u_{i}\left(\mathbf{r}^{\prime}\right) \hat{\mathbf{n}}^{\prime} \times \frac{\mathbf{r}-\mathbf{r}^{\prime}}{\left|\mathbf{r}-\mathbf{r}^{\prime}\right|^{3}} d s\left(\mathbf{r}^{\prime}\right),
$$

where the wedge on the top indicates unit vector.

It is easily shown that if $\mathbf{r} \in G_{i}$, then no contribution is obtained from the singularity at $\mathbf{r}$ since on any little sphere around $\mathbf{r}$,

$$
\hat{\mathbf{n}}^{\prime} \times \frac{\mathbf{r}-\mathbf{r}^{\prime}}{\left|\mathbf{r}-\mathbf{r}^{\prime}\right|^{3}}=\frac{\mathbf{r}-\mathbf{r}^{\prime}}{\left|\mathbf{r}-\mathbf{r}^{\prime}\right|} \times \frac{\mathbf{r}-\mathbf{r}^{\prime}}{\left|\mathbf{r}-\mathbf{r}^{\prime}\right|^{3}}=0 .
$$

Formula (26) is true for $i=1,2, \ldots, n-1$, but for $i=n$, a deeper analysis is necessary. In fact, we have to exclude the singularity from the domain of integration by eliminating from $G_{n}$ a little sphere of radius $\varepsilon>0$ centered at $\mathbf{r}_{0}$ and denote the rest of $G_{n}$ by $G_{n}(\varepsilon)$. In fact, we choose an $\varepsilon>0$ such that

$$
B\left(\mathbf{r}_{0} ; \varepsilon\right)=\left\{\mathbf{r} \in G_{n}:\left|\mathbf{r}-\mathbf{r}_{0}\right| \leq \varepsilon\right\} \subset G_{n}
$$

and let $S\left(\mathbf{r}_{0} ; \varepsilon\right)=\partial B\left(\mathbf{r}_{0} ; \varepsilon\right)$.

Then

$$
G_{n}(\varepsilon)=G_{n}-B\left(\mathbf{r}_{0} ; \varepsilon\right)
$$

and

$$
\partial G_{n}(\varepsilon)=S_{n} \cup S\left(\mathbf{r}_{0} ; \varepsilon\right) .
$$

The electric potential in $G_{n}$ is written as

$$
u_{n}(\mathbf{r})=\frac{1}{4 \pi \sigma_{n}} \mathbf{Q} \cdot \frac{\mathbf{r}-\mathbf{r}_{0}}{\left|\mathbf{r}-\mathbf{r}_{0}\right|^{3}}+w(\mathbf{r}), \quad \mathbf{r} \in G_{n},
$$


where $w$ is a harmonic function and $(26)$ for the $G_{n}(\varepsilon)$ region is written as

$$
\begin{aligned}
& \int_{G_{n}(\varepsilon)} \nabla u_{n}\left(\mathbf{r}^{\prime}\right) \times \frac{\mathbf{r}-\mathbf{r}^{\prime}}{\left|\mathbf{r}-\mathbf{r}^{\prime}\right|^{3}} d \nu\left(\mathbf{r}^{\prime}\right)=\int_{S_{n}} u_{n}\left(\mathbf{r}^{\prime}\right) \hat{\mathbf{n}}^{\prime} \times \frac{\mathbf{r}-\mathbf{r}^{\prime}}{\left|\mathbf{r}-\mathbf{r}^{\prime}\right|^{3}} d s\left(\mathbf{r}^{\prime}\right) \\
& -\frac{\mathbf{Q}}{4 \pi \sigma_{n}} \cdot \int_{S\left(\mathbf{r}_{0} ; \varepsilon\right)} \frac{\mathbf{r}^{\prime}-\mathbf{r}_{0}}{\left|\mathbf{r}^{\prime}-\mathbf{r}_{0}\right|^{3}} \otimes \hat{\mathbf{n}}^{\prime} \times \frac{\mathbf{r}-\mathbf{r}^{\prime}}{\left|\mathbf{r}-\mathbf{r}^{\prime}\right|^{3}} d s\left(\mathbf{r}^{\prime}\right)-\int_{S\left(\mathbf{r}_{0}: \varepsilon\right)} w\left(\mathbf{r}^{\prime}\right) \hat{\mathbf{n}}^{\prime} \times \frac{\mathbf{r}-\mathbf{r}^{\prime}}{\left|\mathbf{r}-\mathbf{r}^{\prime}\right|^{3}} d s\left(\mathbf{r}^{\prime}\right) .
\end{aligned}
$$

The symbol $\otimes$ denotes tensor product. The case where $\mathbf{r} \in G_{n}$ is treated as previously and no contribution from the singularity at $\mathbf{r}$ is obtained. On the other hand, even in the case of $\mathbf{r} \in G_{0}$, the singularity due to the dipole source at $\mathbf{r}_{0}$ is still present. It is easy to see that the integrand of the last integral in $(32)$ is bounded over $B\left(\mathbf{r}_{0}: \varepsilon\right)$ for every $\mathbf{r} \in G_{0}$ and therefore

$$
\lim _{\varepsilon \rightarrow 0} \int_{S\left(\mathbf{r}_{0} ; \varepsilon\right)} w\left(\mathbf{r}^{\prime}\right) \hat{\mathbf{n}}^{\prime} \times \frac{\mathbf{r}-\mathbf{r}^{\prime}}{\left|\mathbf{r}-\mathbf{r}^{\prime}\right|^{3}} d s\left(\mathbf{r}^{\prime}\right)=\mathbf{0} .
$$

Next we investigate the limiting value of the integral

$$
\widetilde{\mathbf{H}}(\varepsilon)=\int_{S\left(\mathbf{r}_{0}: \varepsilon\right)} \frac{\mathbf{r}^{\prime}-\mathbf{r}_{0}}{\left|\mathbf{r}^{\prime}-\mathbf{r}_{0}\right|^{3}} \otimes \hat{\mathbf{n}}^{\prime} \times \frac{\mathbf{r}-\mathbf{r}^{\prime}}{\left|\mathbf{r}-\mathbf{r}^{\prime}\right|^{3}} d s\left(\mathbf{r}^{\prime}\right) .
$$

Introducing a spherical coordinate system centered at $\mathbf{r}_{0}$ via the transformation

$$
\mathbf{r}^{\prime}=\mathbf{r}_{0}+\varepsilon \hat{\mathbf{n}}^{\prime},
$$

we immediately see that

$$
d s\left(\mathbf{r}^{\prime}\right)=\varepsilon^{2} d s\left(\hat{\mathbf{n}}^{\prime}\right)
$$

with

$$
\hat{\mathbf{n}}^{\prime}=\frac{\mathbf{r}^{\prime}-\mathbf{r}_{0}}{\left|\mathbf{r}^{\prime}-\mathbf{r}_{0}\right|}
$$

and

$$
\widetilde{\mathbf{H}}(\varepsilon)=\int_{S^{2}} \hat{\mathbf{n}}^{\prime} \otimes \hat{\mathbf{n}}^{\prime} \times \frac{\mathbf{r}-\mathbf{r}_{0}-\varepsilon \hat{\mathbf{n}}^{\prime}}{\left|\mathbf{r}-\mathbf{r}_{0}-\varepsilon \hat{\mathbf{n}}^{\prime}\right|^{3}} d s\left(\hat{\mathbf{n}}^{\prime}\right),
$$

where $S^{2}$ denotes the unit sphere in $\mathbb{R}^{3}$.

Discretizing the continuous variable $\varepsilon$ by setting

$$
\varepsilon_{n}=\frac{1}{n}, \quad n \geq n_{0}=\left[\frac{1}{\varepsilon}\right]+1
$$

so that the limit as $\varepsilon \rightarrow 0+$ is replaced by the discrete limit as $n \rightarrow \infty$ and using the fact that

$$
\left|\frac{\mathbf{r}-\mathbf{r}_{0}-n^{-1} \hat{\mathbf{n}}^{\prime}}{\left|\mathbf{r}-\mathbf{r}_{0}-n^{-1} \hat{\mathbf{n}}^{\prime}\right|^{3}}\right|=\frac{1}{\left|\mathbf{r}-\mathbf{r}_{0}-n^{-1} \hat{\mathbf{n}}^{\prime}\right|^{2}} \leq \frac{1}{\left(\left|\mathbf{r}-\mathbf{r}_{0}\right|-\varepsilon\right)^{2}}<\frac{1}{d^{2}}
$$

where

$$
d=\min \left\{\left|\mathbf{r}-\mathbf{r}_{s}\right|: \mathbf{r}_{s} \in S\right\}>0
$$


the Lebesgue Dominated Convergence Theorem implies that

$$
\begin{aligned}
\lim _{\varepsilon \rightarrow 0} \widetilde{\mathbf{H}}(\varepsilon) & =\lim _{n \rightarrow \infty} \widetilde{\mathbf{H}}\left(\frac{1}{n}\right)=\int_{S^{2}} \hat{\mathbf{n}}^{\prime} \otimes \hat{\mathbf{n}}^{\prime} \times \lim _{n \rightarrow \infty} \frac{\mathbf{r}-\mathbf{r}_{0}-n^{-1} \hat{\mathbf{n}}^{\prime}}{\left|\mathbf{r}-\mathbf{r}_{0}-n^{-1} \hat{\mathbf{n}}^{\prime}\right|^{3}} d s\left(\hat{\mathbf{n}}^{\prime}\right) \\
& =\int_{S^{2}} \hat{\mathbf{n}}^{\prime} \otimes \hat{\mathbf{n}}^{\prime} \times \frac{\mathbf{r}-\mathbf{r}_{0}}{\left|\mathbf{r}-\mathbf{r}_{0}\right|^{3}} d s\left(\hat{\mathbf{n}}^{\prime}\right)=\int_{S^{2}} \hat{\mathbf{n}}^{\prime} \otimes \hat{\mathbf{n}}^{\prime} d s\left(\hat{\mathbf{n}}^{\prime}\right) \times \frac{\mathbf{r}-\mathbf{r}_{0}}{\left|\mathbf{r}-\mathbf{r}_{0}\right|^{3}} .
\end{aligned}
$$

A straightforward application of Gauss's theorem now implies that

$$
\int_{S^{2}} \hat{\mathbf{n}}^{\prime} \otimes \hat{\mathbf{n}}^{\prime} d s\left(\hat{\mathbf{n}}^{\prime}\right)=\int_{S^{2}} \hat{\mathbf{r}} \otimes \hat{\mathbf{r}} d s(\hat{\mathbf{r}})=\int_{|\mathbf{r}| \leq 1} \nabla \otimes \mathbf{r} d \nu(\mathbf{r})=\tilde{\mathbf{I}} \int_{|\mathbf{r}| \leq 1} d \nu(\mathbf{r})=\frac{4 \pi}{3} \tilde{\mathbf{I}}
$$

where $\tilde{\mathbf{I}}$ denotes the identity dyadic.

In view of the above analysis, it is obvious that

$$
\begin{aligned}
& \int_{G_{n}} \nabla u_{n}\left(\mathbf{r}^{\prime}\right) \times \frac{\mathbf{r}-\mathbf{r}^{\prime}}{\left|\mathbf{r}-\mathbf{r}^{\prime}\right|^{3}} d \nu\left(\mathbf{r}^{\prime}\right) \\
& =\lim _{\varepsilon \rightarrow 0}\left[\int_{G_{n}(\varepsilon)} \nabla u_{n}\left(\mathbf{r}^{\prime}\right) \times \frac{\mathbf{r}-\mathbf{r}^{\prime}}{\left|\mathbf{r}-\mathbf{r}^{\prime}\right|^{3}} d \nu\left(\mathbf{r}^{\prime}\right)+\int_{B\left(\mathbf{r}_{0}: \varepsilon\right)} \nabla u_{n}\left(\mathbf{r}^{\prime}\right) \times \frac{\mathbf{r}-\mathbf{r}^{\prime}}{\left|\mathbf{r}-\mathbf{r}^{\prime}\right|^{3}} d \nu\left(\mathbf{r}^{\prime}\right)\right] \\
& =\lim _{\varepsilon \rightarrow 0}\left[\int_{S_{n}} u_{n}\left(\mathbf{r}^{\prime}\right) \hat{\mathbf{n}}^{\prime} \times \frac{\mathbf{r}-\mathbf{r}^{\prime}}{\left|\mathbf{r}-\mathbf{r}^{\prime}\right|^{3}} d s\left(\mathbf{r}^{\prime}\right)-\int_{S\left(\mathbf{r}_{0}: \varepsilon\right)} u_{n}\left(\mathbf{r}^{\prime}\right) \hat{\mathbf{n}}^{\prime} \times \frac{\mathbf{r}-\mathbf{r}^{\prime}}{\left|\mathbf{r}-\mathbf{r}^{\prime}\right|^{3}} d s\left(\mathbf{r}^{\prime}\right)\right. \\
& \left.\quad+\int_{B\left(\mathbf{r}_{0} ; \varepsilon\right)} \nabla u_{n}\left(\mathbf{r}^{\prime}\right) \times \frac{\mathbf{r}-\mathbf{r}^{\prime}}{\left|\mathbf{r}-\mathbf{r}^{\prime}\right|^{3}} d \nu\left(\mathbf{r}^{\prime}\right)\right] \\
& =\int_{S_{n}} u_{n}\left(\mathbf{r}^{\prime}\right) \hat{\mathbf{n}}^{\prime} \times \frac{\mathbf{r}-\mathbf{r}^{\prime}}{\left|\mathbf{r}-\mathbf{r}^{\prime}\right|^{3}} d s\left(\mathbf{r}^{\prime}\right)-\frac{1}{3 \sigma_{n}} \mathbf{Q} \times \frac{\mathbf{r}-\mathbf{r}_{0}}{\left|\mathbf{r}-\mathbf{r}_{0}\right|^{3}} \\
& \quad+\lim _{\varepsilon \rightarrow 0} \int_{B\left(\mathbf{r}_{0}: \varepsilon\right)} \nabla u_{n}\left(\mathbf{r}^{\prime}\right) \times \frac{\mathbf{r}-\mathbf{r}^{\prime}}{\left|\mathbf{r}-\mathbf{r}^{\prime}\right|^{3}} d \nu\left(\mathbf{r}^{\prime}\right) .
\end{aligned}
$$

Because of the singularity at $\mathbf{r}_{0}$, the last integral in (44) exhibits a hyper-singular behaviour which will be evaluated through regularization $[3,9]$. In fact, we interpret its contribution as a distribution of sources within the ball $B\left(\mathbf{r}_{0} ; \varepsilon\right)$ perturbing, in this way, the location of the source by a small distance $\delta \in(0, \varepsilon)$. The source is then located at the point $\overline{\mathbf{r}}=\mathbf{r}^{\prime}-\mathbf{r}_{0}$. That leads to the perturbed internal electric potential

$$
u_{n}^{\delta}(\overline{\mathbf{r}})=-\frac{1}{4 \pi \sigma_{n}} \mathbf{Q} \cdot \nabla_{\overline{\mathbf{r}}} \frac{1}{\bar{r}+\delta}=\frac{1}{4 \pi \sigma_{n}} \mathbf{Q} \cdot \frac{\overline{\mathbf{r}}}{\bar{r}(\bar{r}+\delta)^{2}},
$$

which implies that

$$
\nabla_{\overline{\mathbf{r}}} u_{n}^{\delta}(\overline{\mathbf{r}})=\frac{1}{4 \pi \sigma_{n}} \mathbf{Q} \cdot\left[\frac{\tilde{\mathbf{I}}}{\bar{r}(\bar{r}+\delta)^{2}}-\frac{3 \bar{r}+\delta}{\bar{r}(\bar{r}+\delta)^{3}} \hat{\overline{\mathbf{r}}} \otimes \hat{\overline{\mathbf{r}}}\right]
$$

Obviously, as $\varepsilon \rightarrow 0, \delta \rightarrow 0$ and formula (43) as well as the limit

$$
\lim _{\varepsilon \rightarrow 0} \frac{\mathbf{r}-\mathbf{r}_{0}-\overline{\mathbf{r}}}{\left|\mathbf{r}-\mathbf{r}_{0}-\overline{\mathbf{r}}\right|^{3}}=\frac{\mathbf{r}-\mathbf{r}_{0}}{\left|\mathbf{r}-\mathbf{r}_{0}\right|^{3}}
$$


imply that

$$
\begin{aligned}
& \lim _{\varepsilon \rightarrow 0} \int_{B\left(\mathbf{r}_{0} ; \varepsilon\right)} \nabla u_{n}\left(\mathbf{r}^{\prime}\right) \times \frac{\mathbf{r}-\mathbf{r}^{\prime}}{\left|\mathbf{r}-\mathbf{r}^{\prime}\right|^{3}} d \nu\left(\mathbf{r}^{\prime}\right) \\
& \quad=\lim _{\varepsilon \rightarrow 0} \frac{\mathbf{Q}}{4 \pi \sigma_{n}} \cdot \int_{0}^{\varepsilon}\left[\frac{\bar{r}}{(\bar{r}+\delta)^{2}} \int_{S^{2}} \tilde{\mathbf{I}} d s(\hat{\overline{\mathbf{r}}})-\frac{\bar{r}(3 \bar{r}+\delta)}{(\bar{r}+\delta)^{3}} \int_{S^{2}} \hat{\overline{\mathbf{r}}} \otimes \hat{\overline{\mathbf{r}}} d s(\hat{\overline{\mathbf{r}}})\right] d \bar{r} \times \frac{\mathbf{r}-\mathbf{r}_{0}}{\left|\mathbf{r}-\mathbf{r}_{0}\right|^{3}} \\
& \quad=\frac{\mathbf{Q}}{3 \sigma_{n}} \times \frac{\mathbf{r}-\mathbf{r}_{0}}{\left|\mathbf{r}-\mathbf{r}_{0}\right|^{3}} \lim _{\varepsilon \rightarrow 0} \int_{0}^{\varepsilon} \frac{2 \delta \bar{r}}{(\bar{r}+\delta)^{3}} d \bar{r}=\frac{\mathbf{Q}}{3 \sigma_{n}} \times \frac{\mathbf{r}-\mathbf{r}_{0}}{\left|\mathbf{r}-\mathbf{r}_{0}\right|^{3}}\left[1-\lim _{\varepsilon \rightarrow 0} \int_{0}^{\varepsilon} \frac{2 \delta \varepsilon+\delta^{2}}{(\varepsilon+\delta)^{2}}\right] .
\end{aligned}
$$

Choosing

$$
\delta=\varepsilon^{\alpha}, \quad \alpha>1
$$

so that for $\varepsilon \in(0,1)$ it is always true that $\delta \in(0, \varepsilon)$, we see that

$$
\lim _{\varepsilon \rightarrow 0} \frac{2 \delta \varepsilon+\delta^{2}}{(\varepsilon+\delta)^{2}}=\lim _{\varepsilon \rightarrow 0} \frac{2 \varepsilon^{\alpha-1}+\varepsilon^{2(\alpha-1)}}{\left(1+\varepsilon^{\alpha-1}\right)^{2}}=0 .
$$

Hence, in (44) the limiting contribution from the surface $S\left(\mathbf{r}_{0} ; \varepsilon\right)$ and the contribution from the point singularity at $\mathbf{r}_{0}$ cancel each other, leaving us with the identity

$$
\int_{G_{n}} \nabla u_{n}\left(\mathbf{r}^{\prime}\right) \times \frac{\mathbf{r}-\mathbf{r}^{\prime}}{\left|\mathbf{r}-\mathbf{r}^{\prime}\right|^{3}} d \nu\left(\mathbf{r}^{\prime}\right)=\int_{S_{n}} u_{n}\left(\mathbf{r}^{\prime}\right) \hat{\mathbf{n}} \times \frac{\mathbf{r}-\mathbf{r}^{\prime}}{\left|\mathbf{r}-\mathbf{r}^{\prime}\right|^{3}} d s\left(\mathbf{r}^{\prime}\right) .
$$

We observe, though, that in the limit as $\varepsilon \rightarrow 0$ the flux that crosses the surface $S\left(\mathbf{r}_{0} ; \varepsilon\right)$ is equal to one third of the contribution of the primary dipole to the magnetic field, while the concentrated point source contributes minus one third of the same quantity.

Substituting (26) for each $i=1,2, \ldots n-1$ as well as (51) for $i=n$ into the expression (14) and following classical arguments, we are lead to the following form of the Geselowitz formula:

$$
\begin{aligned}
\mathbf{B}(\mathbf{r})=\frac{\mu_{0}}{4 \pi} & \mathbf{Q} \times \frac{\mathbf{r}-\mathbf{r}_{0}}{\left|\mathbf{r}-\mathbf{r}_{0}\right|^{3}} \\
& -\frac{\mu_{0}}{4 \pi} \sum_{\substack{i, j=1 \\
i \neq j}}^{n}\left(\sigma_{i}-\sigma_{j}\right) \int_{S_{i, j}} u_{i}\left(\mathbf{r}^{\prime}\right) \hat{\mathbf{n}}_{i j}^{\prime} \times \frac{\mathbf{r}-\mathbf{r}^{\prime}}{\left|\mathbf{r}-\mathbf{r}^{\prime}\right|^{3}} d s\left(\mathbf{r}^{\prime}\right) \\
& -\frac{\mu_{0}}{4 \pi} \sum_{i=1}^{n} \sigma_{i} \int_{S_{i 0}} u_{i}\left(\mathbf{r}^{\prime}\right) \hat{\mathbf{n}}^{\prime} \times \frac{\mathbf{r}-\mathbf{r}^{\prime}}{\left|\mathbf{r}-\mathbf{r}^{\prime}\right|^{3}} d s\left(\mathbf{r}^{\prime}\right) .
\end{aligned}
$$

In particular, for a single isotropic and homogeneous conductor with conductivity $\sigma$, the Geselowitz formula reads

$$
\mathbf{B}(\mathbf{r})=\frac{\mu_{0}}{4 \pi} \mathbf{Q} \times \frac{\mathbf{r}-\mathbf{r}_{0}}{\left|\mathbf{r}-\mathbf{r}_{0}\right|^{3}}-\frac{\mu_{0} \sigma}{4 \pi} \int_{S} u\left(\mathbf{r}^{\prime}\right) \hat{\mathbf{n}}^{\prime} \times \frac{\mathbf{r}-\mathbf{r}^{\prime}}{\left|\mathbf{r}-\mathbf{r}^{\prime}\right|^{3}} d s\left(\mathbf{r}^{\prime}\right),
$$

where $S$ is the boundary of the conductor and $\mathbf{r} \in G_{0}$. 
A similar sequence of arguments leads to the following Geselowitz formula [4] for the electric potential:

$$
\begin{aligned}
\sigma_{\kappa} u_{\kappa}(\mathbf{r})= & \frac{1}{4 \pi} \mathbf{Q} \cdot \frac{\mathbf{r}-\mathbf{r}_{0}}{\left|\mathbf{r}-\mathbf{r}_{0}\right|^{3}} \\
& -\frac{1}{4 \pi} \sum_{\substack{i, j=1 \\
i \neq j}}^{n}\left(\sigma_{i}-\sigma_{j}\right) \int_{S_{i j}} u_{i j}\left(\mathbf{r}^{\prime}\right) \hat{\mathbf{n}}_{i j}^{\prime} \cdot \frac{\mathbf{r}-\mathbf{r}^{\prime}}{\left|\mathbf{r}-\mathbf{r}^{\prime}\right|^{3}} d s\left(\mathbf{r}^{\prime}\right) \\
& -\frac{1}{4 \pi} \sum_{i=1}^{n} \sigma_{i} \int_{S_{i 0}} u_{i}\left(\mathbf{r}^{\prime}\right) \hat{\mathbf{n}}^{\prime} \cdot \frac{\mathbf{r}-\mathbf{r}^{\prime}}{\left|\mathbf{r}-\mathbf{r}^{\prime}\right|^{3}} d s\left(\mathbf{r}^{\prime}\right)
\end{aligned}
$$

for $\mathbf{r} \in G_{\kappa}$ and $\kappa=1,2, \ldots, n$.

From the theory of Magnetostatics we know that the dipole term in the multipole expansion of the magnetic field outside a bounded volume conductor vanishes.

Indeed, if we define the support of $\mathbf{J}$ by

$$
V=\operatorname{supp} \mathbf{J}
$$

and $S_{R}$ denotes a sphere that contains $V$ in its interior, then Eq. (8) implies that

$$
\nabla \cdot \mathbf{J}(\mathbf{r})=0
$$

which yields the identity

$$
\nabla \cdot(\mathbf{J} \otimes \mathbf{r})=(\nabla \cdot \mathbf{J}) \mathbf{r}+\mathbf{J} \cdot \nabla \otimes \mathbf{r}=\mathbf{J} .
$$

Ampère's law (13) then provides

$$
\begin{aligned}
\mathbf{B}(\mathbf{r}) & =\frac{\mu_{0}}{4 \pi} \int_{V} \mathbf{J}\left(\mathbf{r}^{\prime}\right) \times \frac{\mathbf{r}-\mathbf{r}^{\prime}}{\left|\mathbf{r}-\mathbf{r}^{\prime}\right|^{3}} d \nu\left(\mathbf{r}^{\prime}\right) \\
& =\frac{\mu_{0}}{4 \pi} \nabla_{\mathbf{r}} \times \int_{V} \frac{\mathbf{J}\left(\mathbf{r}^{\prime}\right)}{\left|\mathbf{r}-\mathbf{r}^{\prime}\right|} d \nu\left(\mathbf{r}^{\prime}\right) \\
& =\frac{\mu_{0}}{4 \pi} \nabla_{\mathbf{r}} \times\left[\frac{1}{r} \int_{V} \mathbf{J}\left(\mathbf{r}^{\prime}\right) d \nu\left(\mathbf{r}^{\prime}\right)+O\left(\frac{1}{r^{2}}\right)\right] d \nu\left(\mathbf{r}^{\prime}\right) \\
& =-\frac{\mu_{0}}{4 \pi} \frac{\mathbf{r}}{r^{3}} \times \int_{V} \nabla_{\mathbf{r}^{\prime}} \cdot\left(\mathbf{J}\left(\mathbf{r}^{\prime}\right) \otimes \mathbf{r}^{\prime}\right) d \nu\left(\mathbf{r}^{\prime}\right)+O\left(\frac{1}{r^{3}}\right) \\
& =-\frac{\mu_{0}}{4 \pi} \frac{\mathbf{r}}{r^{3}} \times \int_{S_{R}} \hat{\mathbf{n}}^{\prime} \cdot \mathbf{J}\left(\mathbf{r}^{\prime}\right) \otimes \mathbf{r}^{\prime} d s\left(\mathbf{r}^{\prime}\right)+O\left(\frac{1}{r^{3}}\right) \\
& =O\left(\frac{1}{r^{3}}\right),
\end{aligned}
$$

where Gauss's theorem in the ball interior to $S_{R}$ as well as (55) has been used. Note that in applying Gauss's theorem the same analytical arguments as before have to be used if concentrated currents within the support $V$ are involved. Equation (58) proves that the multipole expansion of $\mathbf{B}$ contains no dipole term. This result is equivalent to the non-existence of magnetic monopoles. 
4. Multipole expansion. In order to obtain a complete multipole expansion of the field $\mathbf{B}$ as it is given by (53) we introduce the Polyadic notation

$$
\begin{gathered}
\bigotimes_{i=1}^{n} \mathbf{a}_{i}=\mathbf{a}_{1} \otimes \mathbf{a}_{2} \otimes \cdots \otimes \mathbf{a}_{n} \\
{\left[\bigotimes_{i=1}^{n} \mathbf{a}_{i}\right] \text { (II) }\left[\bigotimes_{i=1}^{n} \mathbf{b}_{i}\right]=\prod_{i=1}^{n}\left(\mathbf{a}_{i} \cdot \mathbf{b}_{i}\right)} \\
{\left[\bigotimes_{i=1}^{n+1} \mathbf{a}_{i}\right] \stackrel{\text { (II) }}{n}\left[\bigotimes_{i=1}^{n+1} \mathbf{b}_{i}\right]=\prod_{i=1}^{n}\left(\mathbf{a}_{i} \cdot \mathbf{b}_{i}\right)\left(\mathbf{a}_{n+1} \times \mathbf{b}_{n+1}\right) .}
\end{gathered}
$$

which provide the Taylor expansion

$$
\frac{1}{\left|\mathbf{r}-\mathbf{r}_{0}\right|}=\sum_{n=0}^{\infty} \frac{(-1)^{n}}{n !}\left(\mathbf{r}_{0} \cdot \nabla_{\mathbf{r}}\right)^{n} \frac{1}{r}=\sum_{n=0}^{\infty} \frac{(-1)^{n}}{n !}\left(\bigotimes_{i=1}^{n} \mathbf{r}_{0}\right) \mathrm{I}\left(\bigotimes_{i=1}^{n} \nabla\right) \frac{1}{r}
$$

where the formula

$$
\left.\bigotimes_{i=1}^{n} \nabla_{\mathbf{r}} \frac{1}{\left|\mathbf{r}-\mathbf{r}_{0}\right|}\right|_{\mathbf{r}_{0}=\mathbf{0}}=\bigotimes_{i=1}^{n} \nabla \frac{1}{r}
$$

has been used. Expansion (62) holds true for $r>r_{0}$. The importance of the last expansion in (62) is that it shows the separation between the $\mathbf{r}_{0}$ and the $\mathbf{r}$ dependence.

In interpreting (62) we assime the notation

$$
\bigotimes_{i=1}^{0} \mathbf{a}=1
$$

A corresponding expansion for the dipole field $\left(\mathbf{r}-\mathbf{r}_{0}\right)\left|\mathbf{r}-\mathbf{r}_{0}\right|^{-3}$ can be obtained through the action either of the operator $\left(-\nabla_{\mathbf{r}}\right)$ or of $\nabla_{\mathbf{r}_{0}}$ on $(62)$.

This leads to

$$
\frac{\mathbf{r}-\mathbf{r}_{0}}{\left|\mathbf{r}-\mathbf{r}_{0}\right|^{3}}=\sum_{n=0}^{\infty} \frac{(-1)^{n+1}}{n !}\left(\mathbf{r}_{0} \cdot \nabla\right)^{n} \nabla \frac{1}{r}=\sum_{n=0}^{\infty} \frac{(-1)^{n+1}}{n !}\left(\bigotimes_{i=1}^{n} \mathbf{r}_{0}\right) \cong\left(\bigotimes_{i=1}^{n} \nabla\right) \nabla \frac{1}{r}
$$

In fact for the dipole. the quadrupole, and the octapole term of (65). we obtain

$$
\frac{\mathbf{r}-\mathbf{r}_{0}}{\left|\mathbf{r}-\mathbf{r}_{0}\right|^{3}}=\frac{\hat{\mathbf{r}}}{r^{2}}+\mathbf{r}_{0} \cdot \frac{3 \hat{\mathbf{r}} \otimes \hat{\mathbf{r}}-\tilde{\mathbf{I}}}{r^{3}}-\frac{3}{2 r^{4}} \mathbf{r}_{0} \otimes \mathbf{r}_{0}:[2 \hat{\mathbf{r}} \otimes \tilde{\mathbf{I}}+\tilde{\mathbf{I}} \otimes \hat{\mathbf{r}}-5 \hat{\mathbf{r}} \otimes \hat{\mathbf{r}} \otimes \hat{\mathbf{r}}]+O\left(\frac{1}{r^{5}}\right) \text {. }
$$

If we denote by

$$
\mathbf{q}_{0}=\frac{\mu_{0}}{4 \pi} \mathbf{Q}
$$

the constant dipole moment at $\mathbf{r}_{0}$ and by

$$
\mathbf{q}(\mathbf{r})=-\frac{\mu_{0} \sigma}{4 \pi} u(\mathbf{r}) \hat{\mathbf{n}}
$$

the variable dipole moment at $\mathbf{r} \in S$. we can rewrite (53) as

$$
\mathbf{B}(\mathbf{r})=\mathbf{q}_{0} \times \frac{\mathbf{r}-\mathbf{r}_{0}}{\left|\mathbf{r}-\mathbf{r}_{0}\right|^{3}}+\int_{S} \mathbf{q}\left(\mathbf{r}^{\prime}\right) \times \frac{\mathbf{r}-\mathbf{r}^{\prime}}{\left|\mathbf{r}-\mathbf{r}^{\prime}\right|^{3}} d s\left(\mathbf{r}^{\prime}\right) .
$$


Introducing (65) into (69) we obtain the polyadic multipole expansion

$$
\begin{aligned}
\mathbf{B}(\mathbf{r})= & \sum_{n=0}^{\infty} \frac{(-1)^{n+1}}{n !}\left[\left(\bigotimes_{i=1}^{n} \mathbf{r}_{0}\right) \otimes \mathbf{q}_{0}\right] \stackrel{\mathrm{I}}{\times}\left(\bigotimes_{i=1}^{n+1} \nabla\right) \frac{1}{r} \\
& +\sum_{n=0}^{\infty} \frac{(-1)^{n+1}}{n !}\left[\int_{S}\left(\bigotimes_{i=1}^{n} \mathbf{r}^{\prime}\right) \otimes \mathbf{q}\left(\mathbf{r}^{\prime}\right) d s\left(\mathbf{r}^{\prime}\right)\right] \underset{\times}{\stackrel{\mathrm{In}}{\times}\left(\bigotimes_{i=1}^{n+1} \nabla\right) \frac{1}{r}}
\end{aligned}
$$

At this point we define the polyadic moments of $\mathbf{q}$ as

$$
\begin{aligned}
\mathbb{M}_{0} & =\int_{S} \mathbf{q}(\mathbf{r}) d s(\mathbf{r}) \\
\mathbb{M}_{1} & =\int_{S} \mathbf{r} \otimes \mathbf{q}(\mathbf{r}) d s(\mathbf{r}) \\
\mathbb{M}_{2} & =\int_{S} \mathbf{r} \otimes \mathbf{r} \otimes \mathbf{q}(\mathbf{r}) d s(\mathbf{r})
\end{aligned}
$$

and, in general,

$$
\mathbb{M}_{n}=\int_{S}\left(\bigotimes_{i=1}^{n} \mathbf{r}\right) \otimes \mathbf{q}(\mathbf{r}) d s(\mathbf{r}), \quad n=0,1,2, \ldots
$$

where $\mathbb{M}_{n}$ is a polyadic of order $n+1$.

Then (70) can be rewritten as

$$
\mathbf{B}(\mathbf{r})=\sum_{n=0}^{\infty} \frac{(-1)^{n+1}}{n !}\left[\left(\bigotimes_{i=1}^{n} \mathbf{r}_{0}\right) \otimes \mathbf{q}_{0}+\mathbb{M}_{n}\right] \underset{\times}{\stackrel{\mathrm{n}}{\times}\left(\bigotimes_{i=1}^{n+1} \nabla\right) \frac{1}{r}}
$$

or, in particular,

$$
\begin{aligned}
\mathbf{B}(\mathbf{r})=- & \left(\mathbf{q}_{0}+\mathbb{M}_{0}\right) \times \nabla \frac{1}{r}+\left(\mathbf{r}_{0} \otimes \mathbf{q}_{0}+\mathbb{M}_{1}\right): \nabla \otimes \nabla \frac{1}{r} \\
& -\frac{1}{2}\left(\mathbf{r}_{0} \otimes \mathbf{r}_{0} \otimes \mathbf{q}_{0}+\mathbb{M}_{2}\right):{ }_{\times} \nabla \otimes \nabla \otimes \nabla \frac{1}{r}+O\left(\frac{1}{r^{5}}\right) .
\end{aligned}
$$

Note that (58) implies that

$$
\mathbf{q}_{0}+\mathbb{M}_{0}=\mathbf{0}
$$

or equivalently that

$$
\mathbf{Q}=\int_{S} \sigma u(\mathbf{r}) \hat{\mathbf{n}} d s(\mathbf{r})
$$

Hence, for any surface $S$, the zeroth order moment coincides with the dipole moment of the primary field.

This is easily verified for the sphere if the solution

$$
u(\mathbf{r})=c+\frac{\mathbf{Q} \cdot \hat{\mathbf{r}}}{2 \pi \sigma}\left(\frac{1}{2 r^{2}}+\frac{r}{\alpha^{3}}\right)+\text { higher multipole terms }
$$

with $c$ an arbitrary constant, as well as orthogonality of the surface spherical harmonics, are used. In particular, the leading (quadrupole) term in the expansion (70) of $\mathbf{B}$ is given 
by

$$
\begin{aligned}
\mathbf{B}(\mathbf{r}) & =\left[\mathbf{r}_{0} \otimes \mathbf{q}_{0}+\int_{S} \mathbf{r} \otimes \mathbf{q}(\mathbf{r}) d s(\mathbf{r})\right] \times \frac{3 \hat{\mathbf{r}} \otimes \hat{\mathbf{r}}-\tilde{\mathbf{I}}}{r^{3}}+O\left(\frac{1}{r^{4}}\right) \\
& =\frac{\mu_{0}}{4 \pi}\left[\mathbf{r}_{0} \otimes \mathbf{Q}-\sigma \int_{S} u(\mathbf{r}) \mathbf{r} \otimes \hat{\mathbf{n}} d s(\mathbf{r})\right] \times \frac{3 \hat{\mathbf{r}} \otimes \hat{\mathbf{r}}-\tilde{\mathbf{I}}}{r^{3}}+O\left(\frac{1}{r^{4}}\right) .
\end{aligned}
$$

5. The single sphere model. Sarvas [13] considered the case of a single conductive sphere of radius $\alpha$, with an electric dipole in its interior, and he observed that because of the identity

$$
\left(\hat{\mathbf{r}}^{\prime} \times \frac{\mathbf{r}-\mathbf{r}^{\prime}}{\left|\mathbf{r}-\mathbf{r}^{\prime}\right|^{3}}\right) \cdot \hat{\mathbf{r}}=0,
$$

the radial component of the magnetic field $\mathbf{B}$ coincides with the radial component of the primary dipole field $\mathbf{B}_{0}$. In other words, the spherical symmetry forces the contribution of the boundary to live on an orthogonal to $\hat{\mathbf{r}}$ plane. Then he proceeded to evaluate the magnetic field outside the sphere as a consequence of the fact that $\mathbf{B}$ is irrotational there. In fact, he assumed the representation

$$
\mathbf{B}(\mathbf{r})=-\mu_{0} \nabla U(\mathbf{r})
$$

for $|\mathbf{r}|>\alpha$ and he obtained the potential function $U$ by integrating over a ray from the observation point $\mathbf{r}$ to infinity where $U$ vanishes asymptotically. This integration demands only the radial component of $\mathbf{B}$ and therefore only the radial component of $\mathbf{B}_{0}$ is utilized. This program [13] leads to the magnetic potential

$$
U(\mathbf{r})=-\frac{1}{4 \pi F(\mathbf{r})}\left(\mathbf{Q} \times \mathbf{r}_{0}\right) \cdot \mathbf{r}
$$

and therefore to the induction field

$$
\mathbf{B}(\mathbf{r})=\frac{\mu_{0}}{4 \pi F^{2}(\mathbf{r})}\left(\mathbf{Q} \times \mathbf{r}_{0}\right) \cdot[F(\mathbf{r}) \tilde{\mathbf{I}}-\mathbf{r} \otimes \nabla F(\mathbf{r})]
$$

where

$$
F(\mathbf{r})=r\left|\mathbf{r}-\mathbf{r}_{0}\right|^{2}+\left(r^{2}-\mathbf{r} \cdot \mathbf{r}_{0}\right)\left|\mathbf{r}-\mathbf{r}_{0}\right|
$$

and

$$
\nabla F(\mathbf{r})=\left(1+\frac{\left|\mathbf{r}-\mathbf{r}_{0}\right|}{r}\right)\left[r\left(\mathbf{r}-\mathbf{r}_{0}\right)+\left|\mathbf{r}-\mathbf{r}_{0}\right| \mathbf{r}\right]+\left(r+\frac{r^{2}-\mathbf{r}_{0} \cdot \mathbf{r}}{\left|\mathbf{r}-\mathbf{r}_{0}\right|}\right)\left(\mathbf{r}-\mathbf{r}_{0}\right) .
$$

Note that everything is proportional to the term $\mathbf{Q} \times \mathbf{r}_{0}$ since

$$
\hat{\mathbf{r}} \cdot \mathbf{B}(\mathbf{r})=-\frac{\mu_{0}}{4 \pi} \frac{\left(\mathbf{Q} \times \mathbf{r}_{0}\right) \cdot \hat{\mathbf{r}}}{\left|\mathbf{r}-\mathbf{r}_{0}\right|^{3}}
$$

and therefore this is the only term of $\mathbf{B}$ that survives its radial projection. Furthermore, the radius of the sphere does not enter in the expression for $\mathbf{B}$.

Performing an asymptotic expansion for $r>r_{0}$ we obtain that

$$
\frac{1}{F(\mathbf{r})}=\frac{1}{2 r^{3}}+\frac{\mathbf{r}_{0} \cdot \hat{\mathbf{r}}}{r^{4}}+O\left(\frac{1}{r^{5}}\right)
$$


and

$$
\frac{\nabla F(\mathbf{r})}{F^{2}(\mathbf{r})}=\frac{3 \hat{\mathbf{r}}}{2 r^{4}}+\frac{(5 \hat{\mathbf{r}} \otimes \hat{\mathbf{r}}-\tilde{\mathbf{I}}) \cdot \mathbf{r}_{0}}{r^{5}}+O\left(\frac{1}{r^{6}}\right)
$$

which imply that

$$
\mathbf{B}(\mathbf{r})=\frac{\mu_{0}}{8 \pi} \frac{\mathbf{Q} \times \mathbf{r}_{0}}{r^{3}} \cdot(\tilde{\mathbf{I}}-3 \hat{\mathbf{r}} \otimes \hat{\mathbf{r}})+\frac{\mu_{0}}{4 \pi} \frac{\mathbf{Q} \times \mathbf{r}_{0}}{r^{4}} \cdot(\tilde{\mathbf{I}} \otimes \hat{\mathbf{r}}+\hat{\mathbf{r}} \otimes \tilde{\mathbf{I}}-5 \hat{\mathbf{r}} \otimes \hat{\mathbf{r}} \otimes \hat{\mathbf{r}}) \cdot \mathbf{r}_{0}+O\left(\frac{1}{r^{5}}\right)
$$

A clear view is obtained if we rewrite the expression (53) for the $\mathbf{B}$ field as

$$
\begin{aligned}
\mathbf{B}(\mathbf{r})=\frac{\mu_{0}}{4 \pi} & \frac{\mathbf{Q} \times \mathbf{r}}{\left|\mathbf{r}-\mathbf{r}_{0}\right|^{3}}-\frac{\mu_{0}}{4 \pi} \frac{\mathbf{Q} \times \mathbf{r}_{0}}{\left|\mathbf{r}-\mathbf{r}_{0}\right|^{3}}-\frac{\mu_{0} \sigma}{4 \pi} \int_{S} u\left(\mathbf{r}^{\prime}\right) \frac{\hat{\mathbf{n}}^{\prime} \times \mathbf{r}}{\left|\mathbf{r}-\mathbf{r}^{\prime}\right|^{3}} d s\left(\mathbf{r}^{\prime}\right) \\
& +\frac{\mu_{0} \sigma}{4 \pi} \int_{S} u\left(\mathbf{r}^{\prime}\right) \frac{\hat{\mathbf{n}}^{\prime} \times \mathbf{r}^{\prime}}{\left|\mathbf{r}-\mathbf{r}^{\prime}\right|^{3}} d s\left(\mathbf{r}^{\prime}\right)
\end{aligned}
$$

where the last integral vanishes whenever $S$ is a sphere. Taking the radial component of $\mathbf{B}$, the first and the third term on the right-hand side of (91) are also eliminated and the evaluation of $\mathbf{B}$ is then based solely on the radial component of the term that is proportional to $\mathbf{Q} \times \mathbf{r}_{0}$.

The straightforward procedure that leads to the determination of the magnetic field $\mathbf{B}$ is to solve first the interior problem that provides the electric potential $u$ and then to calculate $\mathbf{B}$ from (91). If we follow this approach and use spectral methods to evaluate $u$, we arrive at

$$
u(\mathbf{r})=c+\frac{\mathbf{Q} \cdot \hat{\mathbf{r}}}{4 \pi \sigma}\left(\frac{1}{r^{2}}+\frac{2 r}{\alpha^{3}}\right)-\frac{\mathbf{Q} \otimes \mathbf{r}_{0}:(\tilde{\mathbf{I}}-3 \hat{\mathbf{r}} \otimes \hat{\mathbf{r}})}{4 \pi \sigma}\left(\frac{1}{r^{3}}+\frac{3 r^{2}}{2 \alpha^{5}}\right)+\ldots,
$$

where the missing terms are multipoles of order higher than three, $\alpha$ is the radius of the sphere, and $c$ is an arbitrary constant.

Considering only the dipole and the quadrupole terms we obtain

$$
\begin{aligned}
& \frac{\mu_{0}}{4 \pi} \frac{\mathbf{Q} \times \mathbf{r}}{\left|\mathbf{r}-\mathbf{r}_{0}\right|^{3}}=\frac{\mu_{0}}{4 \pi} \frac{\mathbf{Q} \times \hat{\mathbf{r}}}{r^{2}}+\frac{3 \mu_{0}}{4 \pi} \frac{\mathbf{Q} \times \hat{\mathbf{r}} \otimes \hat{\mathbf{r}} \cdot \mathbf{r}_{0}}{r^{3}}+O\left(\frac{1}{r^{4}}\right) \\
& -\frac{\mu_{0}}{4 \pi} \frac{\mathbf{Q} \times \mathbf{r}_{0}}{\left|\mathbf{r}-\mathbf{r}_{0}\right|^{3}}=-\frac{\mu_{0}}{4 \pi} \frac{\mathbf{Q} \times \mathbf{r}_{0}}{r^{3}}+O\left(\frac{1}{r^{4}}\right) \\
& -\frac{\mu_{0} \sigma}{4 \pi} \int_{S} u\left(\mathbf{r}^{\prime}\right) \frac{\hat{\mathbf{r}}^{\prime} \times \mathbf{r}}{\left|\mathbf{r}-\mathbf{r}^{\prime}\right|^{3}} d s\left(\mathbf{r}^{\prime}\right) \\
& =-\frac{\mu_{0}}{4 \pi} \frac{\mathbf{Q} \times \hat{\mathbf{r}}}{r^{2}}-\frac{3 \mu_{0}}{8 \pi} \frac{\left(\mathbf{Q} \times \mathbf{r}_{0}+\mathbf{r}_{0} \otimes \mathbf{Q}\right) \times \hat{\mathbf{r}}}{r^{3}}+O\left(\frac{1}{r^{4}}\right)
\end{aligned}
$$

and then the cancellation of the dipole terms in (91) is obvious.

Hence, the leading term of the multipole expansion of $\mathbf{B}$ for the sphere is given by the expression

$$
\mathbf{B}(\mathbf{r})=\frac{3 \mu_{0}}{4 \pi} \frac{\mathbf{Q} \times \hat{\mathbf{r}} \otimes \hat{\mathbf{r}} \cdot \mathbf{r}_{0}}{r^{3}}-\frac{\mu_{0}}{4 \pi} \frac{\mathbf{Q} \times \mathbf{r}_{0}}{r^{3}}-\frac{3 \mu_{0}}{8 \pi} \frac{\hat{\mathbf{r}} \cdot\left(\mathbf{Q} \otimes \mathbf{r}_{0}+\mathbf{r}_{0} \otimes \mathbf{Q}\right) \times \hat{\mathbf{r}}}{r^{3}}+O\left(\frac{1}{r^{4}}\right)
$$


which, in view of the identity

$$
\begin{aligned}
(\mathbf{Q} \times \hat{\mathbf{r}})\left(\hat{\mathbf{r}} \cdot \mathbf{r}_{0}\right)-\left(\mathbf{r}_{0} \times \hat{\mathbf{r}}\right)(\hat{\mathbf{r}} \cdot \mathbf{Q}) & =\hat{\mathbf{r}} \times\left[\mathbf{Q} \times\left(\mathbf{r}_{0} \times \hat{\mathbf{r}}\right)+\mathbf{r}_{0} \times(\hat{\mathbf{r}} \times \mathbf{Q})\right] \\
& =-\hat{\mathbf{r}} \times\left[\hat{\mathbf{r}} \times\left(\mathbf{Q} \times \mathbf{r}_{0}\right)\right] \\
& =\left(\mathbf{Q} \times \mathbf{r}_{0}\right)(\hat{\mathbf{r}} \cdot \hat{\mathbf{r}})-\left(\mathbf{Q} \times \mathbf{r}_{0}\right) \cdot \hat{\mathbf{r}} \otimes \hat{\mathbf{r}} \\
& =\left(\mathbf{Q} \times \mathbf{r}_{0}\right) \cdot(\tilde{\mathbf{I}}-\hat{\mathbf{r}} \otimes \hat{\mathbf{r}}) .
\end{aligned}
$$

implies that

$$
\mathbf{B}(\mathbf{r})=\frac{\mu_{0}}{8 \pi r^{3}}\left(\mathbf{Q} \times \mathbf{r}_{0}\right) \cdot(\tilde{\mathbf{I}}-3 \hat{\mathbf{r}} \odot \hat{\mathbf{r}})+O\left(\frac{1}{r^{4}}\right)
$$

and recovers the leading quadrupole term in (90).

The present analysis is greatly influenced by the isotropy of the sphere and the inevitable symmetry it implies. A non-isotropic model of the brain, such as the case of an ellipsoid, would reveal a more realistic intercomnection between the electrochemical activity of the brain and the electromagnetic field it generates.

Acknowledgement. The authors want to express their thanks to Professor A. Kotsiolis for fruitful discussion during the preparation of this manuscript.

\section{REFERENCES}

[1] L. Brand, "Vector and Tensor Analysis". John Wiley \& Sons. New York, 1947

[2] B. N. Cuffin and D. Cohen. "Magnetic Fields of a Dipole in Special Volume Conductor Shapes", IEEE Trans. Biom. Eng., BME-24, pp. 372-381, 1977

[3] I. M. Gel'fand and G. E. Shilov, "Generalized Functions". Vol. I-V. Academic Press, New York, 1964

[4] D. B. Geselowitz, "On Bioelectric Potentials in an Inhomogeneous Volume Conductor". Biophys. J.. 7. pp. 1-11, 1967

[5] D. B. Geselowitz. "On the Magnetic Field Generated Outside an Inhomogeneous Volume Conductor by Internal Current Sources". IEEE Trans. Magn., 6. pp. 346-347, 1970

[6] F. Grynszpan and D. B. Geselowitz, "Model Studies of the Magnetocardiogram". Biophys. J.. 13. pp. 911-925, 1973

[7] M. S. Hämäläinen, R. Hari. R. Ilmoniemi, J. Knuutila. and O. Lounasmaa, "Magnetoencephalography-Theory, Instrumentation, and Application to Noninvasive Studies of the Working Human Brain". Rev. Mod. Phys.. 65, pp. 413-497. 1993

[8] R. J. Ilmoniemi. M. S. Hämäläinen, and J. Knuutila. "The Forward and Inverse Problems in the Spherical Model", pp. 278-282 in Biomagnetics: Applications and Theory, edited by Harold Weinberg. Gerhard Stroink, and Toiro Katila, Pergamon Press, New York, 1985

[9] V. D. Kupradze, "Three-Dimensional Problems of the Mathematical Theory of Elasticity and Thermoelasticity". North-Holland. Amsterdam. 1979

[10] L. D. Landau and E. M. Lifshitz. "Electrodynamics of Continuous Media". Pergamon Press. Oxford. 1960

[11] J. Malmivuo and R. Plonsey, "Bioelectromagnetism", Oxford, Univ. Press, New York. 1995

[12] G. Nolte, T. Fieseler, and G. Curio, "Perturbative Analytical Solutions of the Magnetic Forward Problem for Realistic Volume Conductors", J. Appl. Phys., 89, pp. 2360-2369, 2001

[13] J. Sarvas, "Basic Mathematical and Electromagnetic Concepts of the Biomagnetic Inverse Problem", Phys. Med. Biol., 32, pp. 11-22, 1987 